\title{
Association between Chronic Bronchitis and cognitive impairment in elderly Chinese subjects over 90 years of age
}

\author{
Lingyun Gao ${ }^{1,2}$, Birong Dong ${ }^{2}$, Qiu Kui Hao ${ }^{2}$ \\ ${ }^{1}$ Sichuan Academy of Medical Sciences, Sichuan Provincial People's Hospital, Chengdu, China \\ ${ }^{2}$ West China Hospital, West China School of Medicine, Sichuan University, Chengdu, China \\ Email address: \\ birongdong@163.com (Birong Dong)
}

\section{To cite this article:}

Lingyun Gao, Birong Dong, Qiu Kui Hao. Association between Chronic Bronchitis and Cognitive Impairment in Elderly Chinese Subjects Over 90 Years of Age. American Journal of Health Research. Vol. 2, No. 4, 2014, pp. 203-206.

doi: 10.11648/j.ajhr.20140204.26

\begin{abstract}
Objective: chronic bronchitis may have a key influence on cognitive function, however, the relationship between chronic bronchitis and cognitive impairment in the elderly Chinese population has not been explored. The present study investigated the association between cognitive impairment and chronic bronchitis in elderly Chinese subjects over 90 years of age. Methods: This study comprised data from subjects included in the 2005 Project of Longevity and Ageing in Dujiangyan, China. Subjects were divided into two groups: cognitive impairment group and normal group. Chronic bronchitis data were collected and cognitive function was assessed in all subjects using the Mini-Mental State Examination. Data were presented as mean \pm standard deviation (SD) or number and percentage. All data were computerized for statistical analysis using the Statistical Package for Social Sciences (SPSS) software package, version 11.5 software program. Results: Data from 763 subjects (249 men, 514 women) were included. There was no statistically significant difference in history of chronic bronchitis between the two groups. Education level in the cognitive impairment group was significantly lower than in the normal group. Conclusions: chronic bronchitis were not related to cognitive impairment in elderly Chinese people over 90 years of age.
\end{abstract}

Keywords: Cognitive Impairment, Chronic Bronchitis, Mini-Mental State Examination, Alzheimer's Disease

\section{Introduction}

Cognitive impairment is a frequent feature of Chronic Obstructive Pulmonary Disease (COPD). However, the proportion of patients with COPD with mild cognitive impairment (MCI) is still unknown, and no screening test has been validated to date for detecting MCI in this population.[1] By 2050, 115 million people worldwide are expected to have some form of cognitive impairment, including mild cognitive impairment and Alzheimer's disease, which is the most common form in the aged.[2] Cognition in the elderly ranges from cognitive changes associated with normal ageing to dementia, with a transitional stage of mild cognitive impairment that may indicate early signs of Alzheimer's disease.[3] Studies have suggested that cognitive impairment is related to COPD and chronic bronchitis.[4-5] and chronic bronchitis is considered to be one of the most influential factors relating to cognitive function. [ 6] The primary aim of the present study was to investigate the association between chronic bronchitis and cognitive impairment in elderly (over 90 years of age) Chinese subjects.

\section{Subjects and Methods}

\subsection{Study Population and Study Design}

The present questionnaire-based, crosssectional study was performed between April 2005 and September 2009 in Dujiangyan, Chengdu, China, as part of the Project of Longevity and Ageing in Dujiangyan (PLAD). [7] The study aimed to include all nonagenarians and centenarians in the area of Dujiangyan, although subjects with post-stroke related disease or Parkinson's disease, or who did not complete the MMSE test, were excluded. The study protocol was approved by the research Ethics Committee of Sichuan University. All participants (and their legal proxies) 
provided written informed consent.

\subsection{Data Collection}

Trained medical personnel visited all recruits at their homes for data collection. All data relating to cognitive function, history of chronic bronchitis (including age, sex, education level, cognitive function, history of chronic bronchitis, hypertension, diabetes and other items) were collected. All information was checked and confirmed by family members who usually lived with the participant.

\subsection{Assessment of Cognitive Function}

Cognitive function was assessed using a Mini-Mental State Examination (MMSE). [8] which included 30 items, each having a 1-point score. Any score of 25 points and above (out of 30)indicated normal cognition. Below this scores could indicate severe (9 points or less), moderate (10-20 points) or mild (21-24points) cognitive impairment. In the presentstudy, some items were modified on the basis that the modification would not change the meaning of the test, as described in a previous study.[19] Subjects were categorized as follows: cognitive impairment, scores less than 17; normal, scores between 17 and 30. [ 8-10]

\subsection{Assessment of History of Chronic Bronchitis}

All participants completed a questionnaire relating to chronic bronchitis, including questions on: Do you have the signs of chronic bronchitis (Coughing,Wheezing, feeling short of breath)for over 2 years. These signs can be serious enough to affect your daily activities.

\subsection{Statistical Analyses}

Data were presented as mean \pm standard deviation (SD) or number and percentage. All data were computerized for statistical analysis using the Statistical Package for Social Sciences (SPSS) software package, version 11.5 software program.

Baseline characteristics were analysed using Chi Square $\left(\mathrm{X}^{2}\right)$-test or Fisher's exact test; Multivariate logistic regression analysis was performed using the first item in each category as the reference value. All tests were two tailed and a P-value $\leq 0.05$ was considered statistically significant.

\section{Results}

Table 1. Characteristics of chronic bronchitis in 763 elderly Chinese subjects ( $>90$ years of age) divided into two groups (cognitive impairment or normal).

\begin{tabular}{lllll}
\hline Chronic bronchitis & Total subjects & Cognitive impairment group MMSE $<17$ & Normal group MMSE $\geq \mathbf{1 7}$ & Statistical significance \\
\hline & $\mathrm{N}=763$ & $\mathrm{~N}=436$ & $\mathrm{~N}=327$ & $\mathrm{P}<0.001$ \\
$\mathrm{Y}$ & 249 & $95(21.8)$ & $154(47.1)$ & \\
$\mathrm{N}$ & 514 & $341(78.2)$ & $173(52.9)$ & \\
\hline
\end{tabular}

Data expressed as n (\%) of subjects.

MMSE, Mini-Mental State Examination.

Y,have chronic bronchitis

N,No chronic bronchitis

Table 2. Multivariate logistic regression analysis of sociodemographic data and history of chronic bronchitis in elderly Chinese subjects ( $\geq 90$ years of age) divided into two groups (cognitive impairment or normal).

\begin{tabular}{|c|c|c|c|c|c|}
\hline \multirow{2}{*}{ Characteristic } & \multirow[b]{2}{*}{ B } & \multirow{2}{*}{ SB } & \multirow{2}{*}{ OR } & \multicolumn{2}{|c|}{ 95\% C.I. } \\
\hline & & & & Lower & Upper \\
\hline Demographic Sex, male/female & -0.38 & 0.24 & 0.68 & 0.42 & 1.10 \\
\hline \multicolumn{6}{|l|}{ Age $<95$ years old } \\
\hline Age $\geq 95-<100$ years & -0.40 & 0.22 & 0.67 & 0.43 & 1.04 \\
\hline Age $\geq 100$ years & -0.90 & 0.45 & 0.41 & 0.17 & 1.00 \\
\hline \multicolumn{6}{|l|}{ Education level } \\
\hline \multicolumn{6}{|l|}{ Illiterate } \\
\hline Literate & 1.16 & 0.29 & 3.19 & 1.82 & 5.60 \\
\hline Primary school & 1.44 & 0.43 & 4.23 & 1.83 & 9.77 \\
\hline Junior high school & 1.36 & 0.77 & 3.91 & 0.87 & 17.68 \\
\hline High school and above & 20.98 & 22008.01 & 1.30 & 0 & \\
\hline \multicolumn{6}{|l|}{ Work history } \\
\hline \multicolumn{6}{|l|}{ Farmer } \\
\hline Domestic worker & -0.22 & 0.36 & 0.80 & 0.39 & 1.63 \\
\hline Industrial worker & 0.00 & 0.54 & 1.05 & 0.36 & 3.00 \\
\hline Professional and technical worker & 0.85 & 0.76 & 2.35 & 0.53 & 10.42 \\
\hline Administrative worker & 36.66 & 21077.03 & 8.35 & 0 & \\
\hline Others & -0.08 & 0.46 & 0.92 & 0.37 & 2.29 \\
\hline \multicolumn{6}{|l|}{ Chronic bronchitis } \\
\hline \multicolumn{6}{|l|}{ Yes } \\
\hline No & 0.27 & 0.24 & 1.31 & 0.84 & 2.06 \\
\hline
\end{tabular}

Reference values for multivariate logistic regression analysis in each category were: female; age < 95 years; illiterate; farmer; Yes. OR, Odds Ratio; CI, confidence interval. 
Out of 870 Dujiangyan inhabitants over 90 years of age, 23 males and 31 females were excluded due to post-stroke related disease or Parkinson's disease and 38 males and 15 females were excluded because they did not complete the MMSE test. A total of 763 participants from the PLAD study (249 [32.6\%] males, 514 [67.4\%] females) were included in the present analysis. Subjects were divided into two groups: cognitive impairment group (MMSE score less than 17, $\mathrm{N}=436$ ) and normal group (MMSE

Score over $17, \mathrm{n}=327$ ). In the cognitive impairment group, the mean age of participants was 94.95 years (range, 90-108 years, 341 females and 95 males). In the normal group, mean age was 92.95 years (range, 90-105years, 173 females and 154 males). The mean \pm SD MMSE score was $10.90 \pm 4.13$ in the cognitive impairment group and $20.37 \pm 2.78$ in the normal group.

Education levels in the cognitive impairment group were significantly lower than levels reported in the normal group $(\mathrm{P}<0.05$, overall value): $375(86.0 \%)$ versus $181(55.4 \%)$ were illiterate; $42(9.6 \%)$ versus $90(27.5 \%)$ were literate; 16 $(3.7 \%)$ versus $38(11.6 \%)$ were educated to primary school level; three $(0.7 \%)$ versus $12(3.7 \%)$ were educated to junior high school level; none $(0.0 \%)$ versus six $(1.8 \%)$ were educated to high school level or above (cognitive impairment group versus normal group, respectively). Single factor analysis revealed that there were significantly more farmers in the cognitive impairment group (355 [81.4\%]) compared with the normal group (241 [73.7\%], $\mathrm{P}<0.05)$. There were significantly fewer industrial workers (10 [2.3\%] versus 18 [5.5\%]), professional and technical workers (four $[0.9 \%]$ versus $15[4.6 \%]$ ), and workers who had administrative or other functions (18 [4.1\%] versus 27 [8.3\%]) in the cognitive impairment group compared with the normal group, $(\mathrm{P}<0.05$, single factor analysis $)$. No statistically significant between-group difference was found in relation to those who performed domestic duties (49 [11.2\%] versus 26 [8.0\%]).

The analysis of chronic bronchitis related data included the following participant numbers: 763 participants in this category, Significant association was found between chronic bronchitis and cognitive impairment, $\mathrm{P}<0.001$.

Using multivariate logistic regression analysis, no statistically significant association was found between the two groups in terms of chronic bronchitis (Table 2).

\section{Discussion}

There are many studies relating to the common problem of cognitive impairment in the elderly[ 8,11-13] and it has been reported that about $5 \%$ of people with dementia are $>65$ years of age. Cognitive impairment is a frequent feature of COPD. [ 14] A study included 1425 cognitively normal individuals aged 70 to 89 years, with 5.1 years follow-up, Then author concluted that COPD as a risk factor for MCI and may provide a substrate for early intervention to prevent or delay the onset and progression of MCI, particularly
NA-MCI.[6] Another population-based study thought that with more than 25 years of follow-up, midlife COPD and asthma were associated with an almost two-fold risk of MCI and dementia later in life. Pulmonary diseases diagnosed later in life seemed to have an inverse relationship with cognitive impairment probably reflecting survival bias. [4]

In the present study, subjects were categorized into a cognitive impairment group (MMSE scores $<17$ ) and normal group (MMSE scores between 17 and 30). The MMSE score level was set according to the fact that most participants in a previous PLAD study had no formal education. [ 7-8,10] The main difference between the present study and the original analyses and report on the PLAD study was that the original report focused on minor cognitive impairment, and excluded the normal and the dementia group. [9] The present study found no statistically significant differences in terms of chronic bronchitis $(\mathrm{P}<0.001)$ between the two groups. It has been reported that no statistically significant association was found between the two groups in terms of chronic bronchitis after adjusting for independent variable factors (sex, age, education level and major occupation before 60 years of age). The result was different from that obtained by Singh [6], suggesting that chronic bronchitis may have little or no effect on cognitive function in Chinese elderly people over 90 years of age.

\section{Limitations}

The present study was limited by a number of factors: most of the participants lived in the countryside and this population might not be representative of the urban population; as part of the PLAD study, there might be a survival bias, however, this was an unavoidable study characteristic; the number of participants was relatively small, due to the difficulty of collecting data from very elderly participants in one area.

\section{Conclusion}

In conclusion, decline in cognitive function is a public health concern that is related to serious physical and mental health problems in aged people. Cognitive decline in the elderly is particularly important for the individuals themselves, but also places a heavy burden on family members. Studies into the impact of history of chronic bronchitis on cognitive function are becoming increasingly important, although in the present study chronic bronchitis were not found to be related to cognitive impairment in Chinese elderly people $>90$ years of age.

\section{Acknowledgements}

The authors thank the staff of the Department of Geriatrics Medicine, West China Hospital, Dujiangyan Government and Dujjiangyan People's Hospital, and all participants (as well as their legal proxies) for their great 
contributions. Declaration of conflicting interest The authors declare that there are no conflicts of interest.

\section{Funding}

This work was supported by the Discipline Construction Foundation of Sichuan University, and by grants from the Project of Science and Technology Bureau of Sichuan Province (No. 2006Z09-006-4) and the Construction Fund for Subjects of West China Hospital of Sichuan University (No. XK05001).

\section{References}

[1] Villeneuve S, Pepin V, Rahayel S,et al. Mild cognitive impairment in moderate to severe COPD: a preliminary study. Chest. 2012;142(6):1516-23.

[2] Prince M, Jackson J (eds) Alzheimer's Disease International World Alzheimer Report 2009 Executive Summary. London: Alzheimer's disease International, 2009.

[3] Kelley BJ and Petersen RC. Alzheimer's disease and mild cognitive impairment. Neurol Clin 2007; 25: 577-609.

[4] Rusanen M1, Ngandu T, Laatikainen T,et al.Chronic obstructive pulmonary disease and asthma and the risk of mild cognitive impairment and dementia: a population based CAIDE study. Curr Alzheimer Res. 2013;10(5):549-55.

[5] Singh B, Parsaik AK, Mielke MM,et al. Chronic obstructive pulmonary disease and association with mild cognitive impairment: the Mayo Clinic Study of Aging. Mayo Clin Proc. 2013, 88(11): 1222-30.
[6] Singh B1, Mielke MM2, Parsaik AK,et al.A Prospective Study of Chronic Obstructive Pulmonary Disease and the Risk for Mild Cognitive Impairment. JAMA Neurol. 2014 Mar 17.

[7] Lingyun Gao, Birong Dong, Qiu Kui Hao,et al. Association between cognitive impairment and eating habits in elderly Chinese subjects over 90 years of age. Journal of International Medical Research 2013; 41(4) :1362-1369.

[8] Folstein MF, Folstein SE and McHugh PR. "Mini-mental state". A practical method for grading the cognitive state of patients for the clinician. J Psychiatr Res 1975; 12: 189-198.

[9] Wang Z, Dong B, Zeng G, et al. Is there an association between mild cognitive impairment and dietary pattern in Chinese elderly? Results from a cross-sectional population study. BMC Public Health 2010; 10: 595.

[10] Tombaugh TN and McIntyre NJ. The minimental state examination: a comprehensive review. J Am Geriatr Soc 1992; 40: 922-935.

[11] 11. Fe'art C, Samieri C and Barberger-Gateau P. Mediterranean diet and cognitive function in older adults. Curr Opin Clin Nutr Metab Care 2010; 13: 14-18.

[12] Roberts RO, Geda YE, Cerhan JR, et al. Vegetables, unsaturated fats, moderate alcohol intake, and mild cognitive impairment. Demen Geriatr Cogn Disord 2010; 29: 413-423.

[13] Van Dyk K and Sano M. The impact of nutrition on cognition in the elderly. Neurochem Res 2007; 32: 893-904.

[14] Villeneuve S, Pepin V, Rahayel S,et al. Mild cognitive impairment in moderate to severe COPD: a preliminary study. Chest. 2012;142(6):1516-23. 